\title{
Strategies for commercial vehicle drivetrains 2030
}

Philip Scarth, FPT Motorenforschung AG

This manuscript is not available according to publishing restriction. Thank you for your understanding. 